\title{
Unequal-sphere packing model for the structural arrangement of the well-ordered adsorbate-substrate system
}

\author{
Alexandre Tkatchenko and Nikola Batina \\ Laboratorio de Nanotecnologia e Ingenieria Molecular, Area de Electroquimica, Departamento de Quimica, \\ Av. San Rafael Atlixco No. 186, Col. Vicentina, Del. Iztapalapa, C.P. 09340 Mexico, D.F., Mexico
}

(Received 27 May 2004; published 3 November 2004)

\begin{abstract}
In order to understand the well-ordered adsorbate-substrate systems at atomic level, a method is developed based on the simulation of packing arrangements for layers of unequal spheres, in three-dimensional space. The model, based on geometrical principles, is developed for fcc structure consisting of two hexagonal ordered layers. During simulation, adsorbate spheres were accommodated in different positions, forming a great variety of structures, in dependence of the intersphere distance of the upper layer spheres. Using the average height of the adsorbate layer on the flat substrate as a determinant parameter, several specific structures have been selected as the most probable: $(\sqrt{ } 3 \times \sqrt{ } 3) \mathrm{R} 30^{\circ},(\sqrt{ } 7 \times \sqrt{ } 7) \mathrm{R} 19.1^{\circ}$, and $(3 \times 3)$. Indeed, they correspond to typical accommodations of the iodine adatoms on the $\mathrm{Pt}(111)$ surface, earlier found in experimental studies, which clearly supports the validity of our model. The model developed in our study could completely and satisfactorily describe the accommodation process of the iodine adlayer on the $\operatorname{Pt}(111)$ surface. This methodology could be of great help for interpretation of scanning tunneling microscopy images, better understanding of adlayer structures, and design of adsorbate-substrate systems with exciting properties.
\end{abstract}

DOI: 10.1103/PhysRevB.70.195403

PACS number(s): 68.35.- p, 68.43.Fg

\section{INTRODUCTION}

For many years, the studies of the well-defined surfaces have been concentrated on the adsorbate-substrate systems in the fields of catalysis, ${ }^{1}$ surface science, ${ }^{2,3}$ surface electrochemistry, ${ }^{4-9}$ etc. Nowadays, due to the rapid development of nanotechnology, it is even more important to understand the atomic structure of adsorbate-substrate systems, which could lead us to the development of surfaces with desired properties. ${ }^{10-14}$ Therefore, an approach involving improvement of the presentation of surface characteristics and the development of methods for better and faster understanding of the different surface properties is required. Traditionally, the most primitive way to present structures of the wellordered surface is by using the so-called hard-ball model. In this very simple approach, the hard balls (circles) represent individual atoms. However, such two-dimensional (2D) presentation is rather useful for graphical purpose and it is not a result of the modeling process.

Note that the problem of understanding the packing arrangement of spheres in 3D space is very old, formulated early by Kepler in his famous conjecture in year 1611. It culminated in its solution in 1998 by T. C. Hales ${ }^{15}$ and Sloane. ${ }^{16}$ The problem of random packing of spheres, related to many different phenomena, has been also widely studied by biologists, materials scientists, engineers, chemists, and physicists. ${ }^{17}$

Understanding the atomic arrangement on the surface of the well-ordered adsorbate-substrate layered system is very complex due to restricted atomic positions and selectivity between different atomic registry. The best example of how such systems can be complex is evident from the recent work and studies concerning interpretation of scanning tunneling microscopy (STM) images. ${ }^{18-22}$ Although we could clearly see the position of individual atoms in STM images, the proper interpretation of topographic properties at atomic level requires knowledge of the exact position or atomic registry of each atom on the substrate surface. Therefore, it is obvious that such complex task requires detailed 3D models, which will correspond to the STM way of presentation. As we will show in our study, the problem could be treated as the packing process of unequal spheres.

The main aim of our study is to develop an adequate model and simulation procedure, which could find the most probable conformations of unequal spheres distributed in two layers, defined as substrate and adsorbate. Indeed it is the beginning of our larger effort to develop a set of models suitable for simulation of surface processes and formation of different structures, possibly useful in modern nanotechnology. Namely, a modeling related approach which involves combinatorial surface science could be a crucial step toward the future design of well-defined surfaces with desired properties.

For simplicity, in this attempt to develop our model, we assume that all spheres within the lower layer (substrate) are of identical size and the upper layer consists of larger spheres. The surface of the substrate is taken to be ideally flat, ordered in hexagonal structure as fcc surface. The upper spheres (adsorbate), as a compact layer, were moved in collective mode over the substrate layer. Every movement of the adlayer generates a new arrangement in respect to the substrate, which is characterized by specific parameters in our model. During the simulation, the intersphere distance between the upper spheres was decreased to change the compactness of the adsorbate layer. Based on data analysis, a special algorithm was developed for recognition of the most likely (optimum) positions of the adsorbate spheres on the flat substrate. The optimum position was defined by using the average distance of the adlayer versus substrate. As could be seen from this summary our model is based on a very simple, 
primitive assumption. However, we have been very curious to find if such a simple approach could lead to a satisfactory description of any known system.

Therefore, we relate results of the adsorbate-substrate unequal-sphere modeling to one of the most-studied and most-known systems: atomic structure of the iodine adlayer on the $\mathrm{Pt}(111)$ surface. The result of this comparison is astonishing. Using our model of the unequal spheres we have been able to identify and determine as the most probable, all structural arrangements of the iodine atoms adsorbed at the $\operatorname{Pt}(111)$ surface, previously found in experiments by STM, ${ }^{18,23-30}$ low-energy electron diffraction (LEED), ${ }^{5,31-36}$ and some other techniques. ${ }^{9,37-44}$ In other words, our question, "Up to which level (how far) a system like chemisorbed iodine on the Pt (111) surface can be described in terms of the possible atomic arrangements, by our model based on geometrical principles?" has a simple answer, "completely." This becomes the first model of such a type that could describe the formation of atomic adlayers of this particular system completely and in great detail.

We believe that the approach developed in our study could be of great importance for a better interpretation of STM images, and helpful for the development and design of devices based on the defined arrangement of well-ordered layers in modern nanotechnology.

\section{PROBLEM DEFINITION}

Before we give a formal statement for the problem, several basic definitions are necessary: $p(i)$-Position vector of the center of $i$ th sphere in the substrate; $P(j)$-Position vector of the center of $j$ th sphere in the adsorbate (it suffices to define the adsorbate layer in terms of the plane coordinates only, as the height of each sphere could be derived from them); Height of a sphere in adsorbate layer-The distance between the center of the sphere and the ground (center of the substrate spheres); Surface coverage $(\Theta)$-Ratio of the number of adsorbate spheres to the number of substrate spheres for a defined area; Corrugation-Difference in height between the lowest and the highest adsorbate spheres; and Packing - A configuration of rigid adsorbate spheres in contact with the substrate.

The height of each sphere in the adsorbate layer, at the final position, is determined after the sphere is lowered in the surface-normal direction until it contacts one or more substrate spheres. By definition, the contact is made when the distance between the adsorbate and the substrate spheres reaches the sum of their radii, regardless of the contact direction. However, the adsorbate height is defined in the surface-normal direction.

The formal definition of the problem is as follows: Find packing configurations of two layers of spheres with radius $R_{1}$ and $R_{2}$, respectively, which satisfy a condition $G(F)$, where $F$ is a function which assigns a numeric value to each packing.

For example, in the equal sphere packing problem, the condition $G(F)$ is almost always the maximum packing efficiency, where $F$ is the packing efficiency, which is defined as the percentage of volume occupied by the spheres.

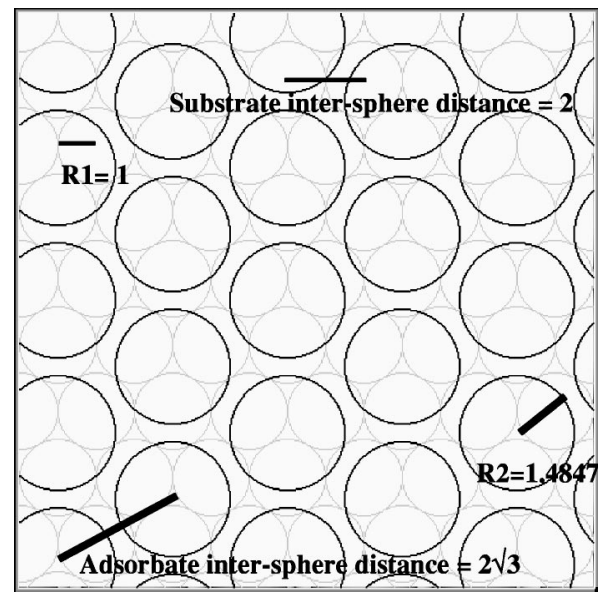

FIG. 1. Illustration of the hexagonally ordered adsorbate and substrate containing spheres of different radii.

In our simulation, different conditions might be chosen to weight the configurations, for example: the lowest adsorbate height average, the lowest corrugation among the adsorbate spheres, the highest corrugation, etc.

In order to define our two-layer system (full monolayers), we apply several conditions. First, the substrate layer is static (flat) with a perfectly defined arrangement (crystalline structure). Second, the adlayer spheres could take different positions in respect to the substrate and have possibility for translation, rotation, and variety of other movements that could lead to a better accommodation on the substrate. Each movement is defined for the complete adlayer, as a collective property for all spheres. In addition, the following definitions are needed to completely define the system. A configuration of adsorbate spheres $P^{\prime}$ is an $\varepsilon$ transformation of another one $P$ if $\left|P^{\prime}(i)-P(i)\right|<\varepsilon$ for each $i$. Consequently, an $\varepsilon$ neighborhood for the adsorbate layer configuration $P$ is defined as the set of all $\varepsilon$ transformations for it. A configuration of the adsorbate spheres $P$ is called a locally minimal configuration if $F(P) \leqslant F\left(P^{\prime}\right)$ for all $P^{\prime}$ in some neighborhood of $P$. Likewise, $P$ is a locally maximal configuration if $F(P) \geqslant F\left(P^{\prime}\right)$ for all $P^{\prime}$ in some neighborhood of $P$.

By means of these definitions, the problem is resolved when all the possible locally optimum packing configurations that satisfy the condition $G(F)$ are found. In other words, the results of the simulation process can be divided in two categories that distinguish two types of packing configurations: those that satisfy $G(F)$ and others that do not.

\section{EXAMPLE STUDY}

In order to verify our model we simulate the structural accommodation of the adsorbate-substrate system with following characteristics:

- Substrate organized in the hexagonal order: (111) fcc type of surface, with $R 1=1$. See light circles with smaller radius, in Fig. 1.

- Adsorbate layer (full monolayer) consisting of $48.47 \%$ bigger spheres $(\mathrm{R} 2=1.4847)$ than the substrate spheres. In principle, the simulation algorithm allows the adsorbate 
spheres to occupy any place on the substrate without any restriction in respect of the structural arrangement.

Note that in terms of the ratio of the spheres radii, and the characteristics of the chosen substrate: (111) fcc face, this system is identical, as we know, to the well-known system of iodine adsorbed at the $\mathrm{Pt}(111)$ substrate, described in great detail in literature. ${ }^{23-44}$ This will allow us to verify our model by direct comparison with structural data obtained by various experimental techniques.

Simulation was carried out in the interval of the surface coverage from $\Theta=0$ to $\Theta=0.4537$, which corresponds to the saturation coverage or a close-packed hexagonal arrangement of adsorbate layer when spheres are touching each other. However we show results of simulation for coverage higher than $\Theta=0.333$ at which, adlayer spheres are arranged in uniform monolayer. We choose this particular coverage using existing experimental data, which will be clarified later in the text. Figure 1 shows such spheres arranged in the structure with 0.333 coverage. In the presented structure, all the adlayer spheres occupy the so-called threefold position on the hexagonal substrate (threefold registry).

This structure is known as $(\sqrt{3} \times \sqrt{3}) \mathrm{R} 30^{\circ}$, and in this particular case, adlayer spheres are located in the threefold position of the substrate. Since all the spheres are located on the same site, the adlayer is, in geometrical sense, completely flat. Any translation along a different axis will give an adlayer with the same characteristics. However, the distance (height) between the substrate and adsorbate spheres will change dramatically. As an example, we would like to mention two other different positions for $(\sqrt{3} \times \sqrt{ } 3) \mathrm{R} 30^{\circ}$ structure: with all the spheres on the atop position and with all the spheres in the twofold position of the substrate. However as we will demonstrate later, those are only a few among many other possible positions. In terms of distances between lower (substrate) spheres and the adsorbate spheres, the minimum distance is obtained when the upper spheres are located in the threefold sites of the (111) surface. On the contrary, the maximum distance is obtained for adsorbate spheres sitting on top of the substrate spheres. Respectively, in many other positions, when the same structure was translated over the substrate layer, the distance between these two extremes was observed.

At this point we introduce a descriptive parameter, in our modeling procedure, defined as adsorbate height average $[A(P)]$. It is the average distance between the adsorbate and substrate layers, calculated by taking into account every distance between the center of adsorbate spheres and the substrate layer regardless of the adsorbate position [see Eq. (1)]

$$
A(P)=\sum_{i=1}^{N} Z\left(P_{i}\right) / N
$$

For example for spheres with $\mathrm{R} 2=1.4847$, the adsorbate height $(Z)$ for a sphere in threefold position is 2.2001 [see Eq. (2)] units normalized to the substrate radius. For a sphere in atop position the value for the adsorbate height is 2.4847 [see Eq. (3)]. Note that in case of $(\sqrt{3} \times \sqrt{3}) \mathrm{R} 30^{\circ}$ structure, all spheres are always in the same position and the average height is equal to the height measured for a single sphere.

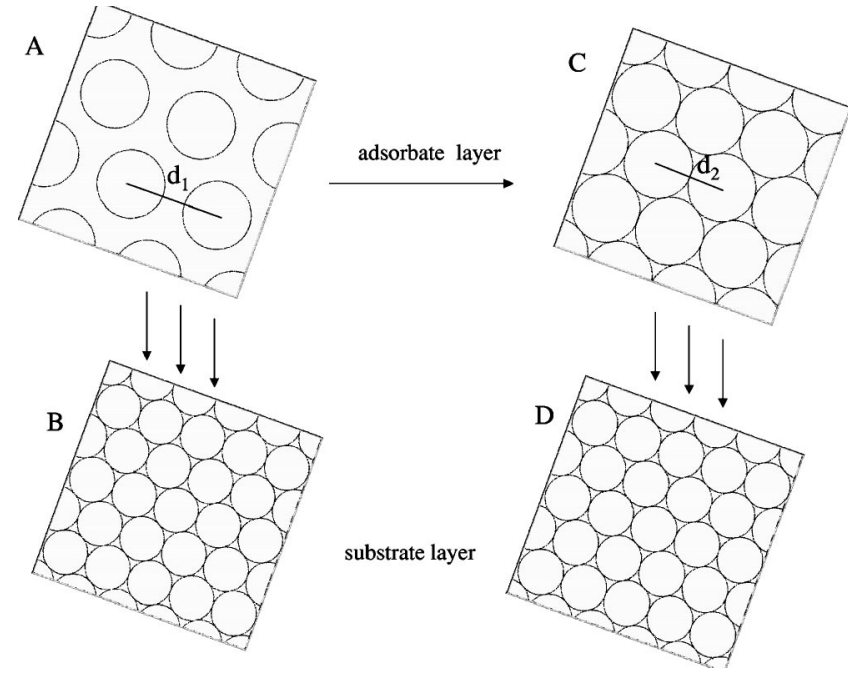

FIG. 2. Scheme of the simulation mechanism, in which adsorbate layers (A and $\mathrm{C}$ ) consisting of spheres of equal diameter but different intersphere distance ( $d_{1}$ and $d_{2}$, respectively) are accommodated on the substrate layer (B and D) with same diameter spheres and same inter-sphere distance.

$$
\begin{gathered}
Z_{\text {threefold }}=\sqrt{\left(R_{1}+R_{2}\right)^{2}-\frac{4}{3} R_{1}^{2}}, \\
Z_{\text {atop }}=R_{1}+R_{2} .
\end{gathered}
$$

However, this ideal situation changes with an increase of the adsorbate coverage. Within our simulation, the adlayer coverage was changed by decreasing the intersphere distance, but keeping the sphere radii constant. It results in the increase of the adsorbate height or distance between upper and lower layers of spheres. The adlayer coverage was increased during simulation process by very small steps, about $1 \%$ of the complete interval range. The illustration of this process can be observed in Fig. 2. With each increment, the adlayer spheres were accommodated on the substrate layer by translating or rotating in all the possible directions. However, during this combinatorial process, the hexagonal order of the adsorbate and the substrate layers was kept fixed. As a result of our simulation, for each intersphere distance, we obtain a specific structural arrangement of the adsorbate spheres with the minimum and the maximum average height (minimum and maximum distance from the substrate layer).

Using our model of unequal-sphere packing we were able to calculate (quantitative presentation) many different parameters: distance between the upper and lower layer of spheres, surface roughness, structure and symmetry characteristics of both layers, geometrical position, and adsorbate-substrate registry. In order to understand the basic principles of the sphere arrangements we analyzed all of these parameters plotting their functional dependence on the different adsorbate layer transformations.

Figure 3 shows the plot of minimum average height between the adsorbate and the substrate layers as a function of the adsorbate (upper layer) intersphere distance, along with the STM mode presentation of several minimum average 


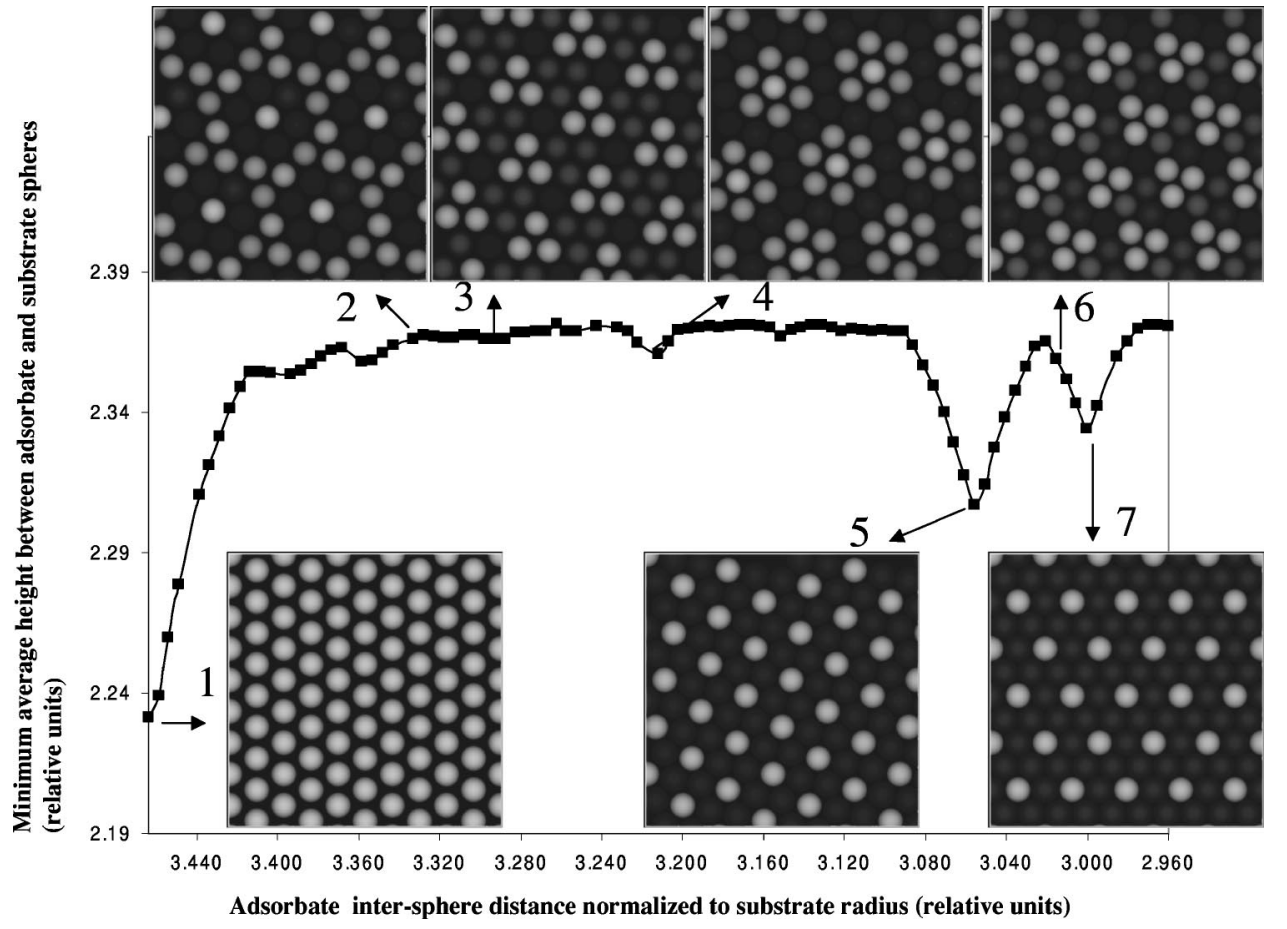

FIG. 3. Plot of minimum average height between the adsorbate and substrate layers versus adsorbate intersphere distance with corresponding images of particular structures, generated by ALSA software. structures. We see a very complex pattern, which could not be explained by a simple function. Each point presented in the graph is related to a specific adsorbate structure that is formed and defined by its intersphere distance. Note that in this particular graph only the structure with the minimum average height is presented for each intersphere distance. In order to assure the precision of our simulation, the minimum structures were checked using a heuristic algorithm.

Looking at the graph presented in Fig. 3, one could see that some of the sphere arrangements are on average much closer to the substrate layer than others. In the simulated function those closer structures are notable as pronounced minima, and marked as points: 1,5 , and 7 . In order to describe structural characteristics of those "closer" structures in an easy and acceptable way, the presentation related to the STM images was used. Note that all spheres presented in Fig. 3 belong to the adlayer (upper layer). Different colors indicate that some adsorbate spheres are up (bright) and another down (dark), but within the same layer. Theoretical STM-like images were generated by ALSA software package developed in our laboratory. ALSA is an acronym for a computational package called "atomic level surface assembler" based on a hard-ball model, which can graphically present surface layers in the way similar to STM images in the Linux environment. Note, in Fig. 3, besides these three arrangements that are very close to the substrate, we also present a few others (marked as: 2, 3, 4, and 6), which are in general further from the substrate surface.

Besides differences in the average height between the adsorbate and the substrate layers, we also noticed, as could be clearly seen in Fig. 3, that structural arrangements in positions: 1,5 , and 7 , posses simpler periodicity.

In order to characterize each of the observed structures we also show them in different modes [Figs. 4(a) and 4(b)]. In these figures we present adlayer spheres and substrate spheres in "transparent" mode, in which the registry of each sphere could be identified. The substrate is presented in the background by lighter circles. It is interesting to note from these images the method of simulation. Substrate and adsorbate spheres posses characteristic diameters, which do not change throughout the simulation process. Comparing the images 1 on Fig.4(a) and 7 on Fig. 4(b) one could see that adsorbate spheres accommodate in a more compact adlayer with smaller intersphere distance. It is clearly seen from these figures that our simulation involves only two layers: substrate (lower smaller circles) and adsorbate (upper larger circles), without differentiation of fcc and hcp threefold sites. Results of this analysis are presented in Table I.

In short, from these results one could see that adsorbate spheres with diameter 1.48 times larger than substrate, forming a hexagonal adlayer on the hexagonal substrate can organize in many different structural arrangements, among which three specific structures: $(\sqrt{ } 3 \times \sqrt{3}) \mathrm{R} 30^{\circ}, \quad(\sqrt{ } 7$ $\times \sqrt{ } 7) \mathrm{R} 19.1^{\circ}$, and $(3 \times 3)$, are distinguished as those closer to the substrate layer. By using the minimum average height between the adsorbate and the substrate, as an identifying parameter in the adlayer stability, we could say that it is the most probable that exactly those three structures will be formed.

At this point it is very important to make correlation with a real system involving atomic adsorbate-substrate layers. As we mentioned before, basic geometrical parameters used in our modeling such as the diameter ratio and hexagonal symmetry for substrate and adsorbate, are very close to the real system of the iodine adlayer formed at the Pt(111) surface. Regarding to numerous experimental data, it is well known that iodine on the $\mathrm{Pt}(111)$ forms well-ordered adlayers with the following structures: $(\sqrt{ } 3 \times \sqrt{ } 3) \mathrm{R} 30^{\circ},(\sqrt{ } 7 \times \sqrt{ } 7) \mathrm{R} 19.1^{\circ}$, and $(3 \times 3){ }^{23-44}$ This is in excellent agreement with data obtained by our model of the unequal-sphere packing. 


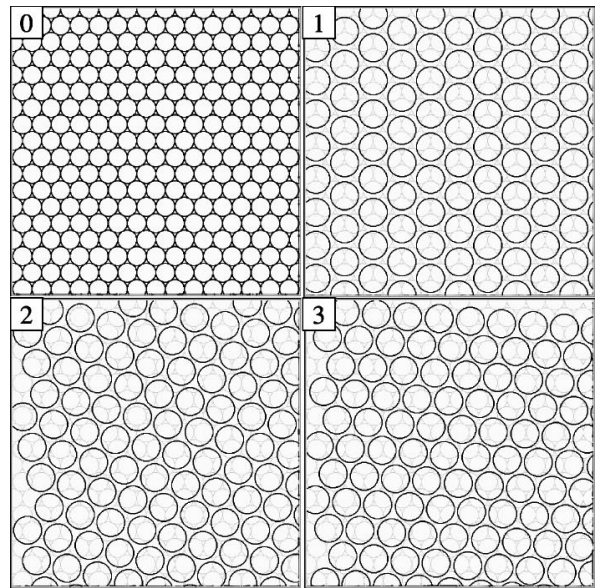

(a)

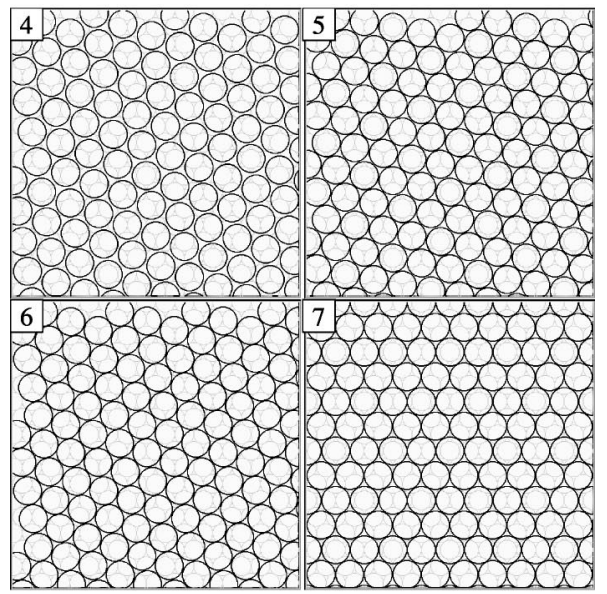

(b)

FIG. 4. Transparent mode presentation of several structures obtained by unequal-sphere packing simulation characterized by data presented in Table I.

There are several important conclusions that could be drawn from this comparison and the obtained agreement.

First is the startling fact that the structure of the iodine adlayer on $\operatorname{Pt}(111)$ can be explained by the unequal-sphere packing simulation based on geometrical principles. It implies that iodine atoms assemble on the $\mathrm{Pt}(111)$ surface in the same way as a group of independent spheres, accommodating on the substrate by geometrical constraints. This result is amazing considering that iodine on $\operatorname{Pt}(111)$ is known to be a chemisorption system, for which many other factors are supposed to contribute to accommodation of the adsorbate atoms on the surface (like fcc-hcp sites, adsorbate-adsorbate interactions, etc.). In our simulation, the threefold sites are equivalent and adsorbate-adsorbate interactions are not taken into account, despite this apparent weakness the simulation shows the formation of three characteristic structures for iodine on $\operatorname{Pt}(111)$ as in real experiments. Therefore the graph presented in Fig. 3 is so far the best function which describes the accommodation of the iodine adatoms on the $\operatorname{Pt}(111)$ surface. Note, once again, that the graph is constructed by simply plotting the minimum average adsorbate-substrate height versus adsorbate intersphere distance. Taking into account that experimentally observed structures mentioned above, for iodine adsorbate on $\operatorname{Pt}(111)$ surface are the most stable ones. In relation to our model it means that for this particular system, the most stable adlayer structures are those which are the closest to the substrate.

Along the same line, we would like to comment the appearance of another structure, which was observed for iodine on $\mathrm{Pt}(111)$ in different experiments and reported in the literature. It is the so-called $(3 \times 3)$-asym structure. ${ }^{24-37,41,42} \mathrm{We}$ believe that our model based on geometrical principles can also explain the appearance of this particular structure. Note that the arrangement of $(3 \times 3)$-asym iodine adlayer on $\operatorname{Pt}(111)$ has been described in many papers, so far. The main characteristics and difference from $(3 \times 3)$-sym adlayer are in the registry of the adsorbed adatoms, while the hexagonal symmetry, the interatomic distances and the adsorbate coverage are preserved in both structures. In $(3 \times 3)$-sym adlayer, six equal bridge positioned neighbors surround each atop iodine atom. In case of $(3 \times 3)$-asym, each threefold iodine atoms is surrounded by six neighboring adatoms positioned at a higher level. ${ }^{24-37}$

In order to understand the conditions for appearance and differences between $(3 \times 3)$-sym and $(3 \times 3)$-asym structures we employ our model, looking for a full range of possible

TABLE I. Parameters relevant for characterization of several structures selected from the graph on Fig. 2.

\begin{tabular}{llccc}
\hline \hline Number & \multicolumn{1}{c}{$\begin{array}{c}\text { Adsorbate inter- } \\
\text { sphere distance } \\
\text { normalized to } \\
\text { substrate radius }\end{array}$} & $\begin{array}{c}\text { Adsorbate } \\
\text { coverage }\end{array}$ & $\begin{array}{c}\text { Average height } \\
\text { between adsorbate and } \\
\text { substrate spheres }\end{array}$ \\
\hline 1 & $(\sqrt{ } 3 \times \sqrt{ } 3) \mathrm{R} 30^{\circ}$ with & 3.4641 & 0.3333 & 2.2001 \\
2 & threefold registry & & & \\
3 & rot-hex with $\alpha=21.8^{\circ}$ & 3.3248 & 0.3611 & 2.3650 \\
4 & rot-hex with $\alpha=4.3^{\circ}$ & 3.2958 & 0.3671 & 2.3611 \\
5 & rot-hex with $\alpha=23.4^{\circ}$ & 3.2146 & 0.3860 & 2.3572 \\
6 & $(\sqrt{ } 7 \times \sqrt{ } 7) \mathrm{R} 19.1^{\circ}$ sym & 3.0548 & 0.4263 & 2.2961 \\
7 & rot-hex with $\alpha=19.1^{\circ}$ & 3.0232 & 0.4360 & 2.3614 \\
\hline \hline & $(3 \times 3)$-sym & 3.0000 & 0.4444 & 2.3271 \\
\hline
\end{tabular}




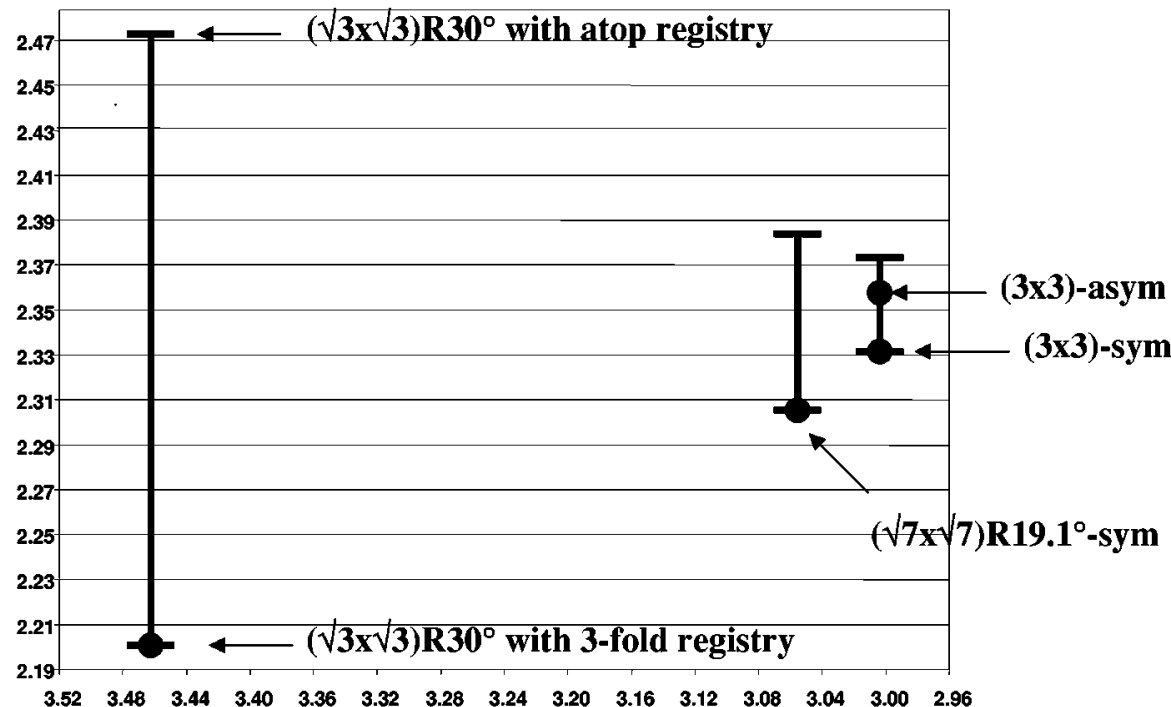

FIG. 5. Plot of a full range of average distance between adsorbate and substrate layers versus adsorbate intersphere distance for three typical structures: $\quad(\sqrt{ } 3$ $\times \sqrt{3}) \mathrm{R} 30^{\circ},(\sqrt{ } 7 \times \sqrt{ } 7) \mathrm{R} 19.1^{\circ}$, and $(3 \times 3)$, due to different adsorbate sphere registry.

\section{Adsorbate inter-sphere distance normalized to substrate radius (relative units)}

structures, when adlayer atoms (in model spheres) are collectively shifting on the substrate layer. The simulation shows the existence of many different structures, which have a distinct value of the average adsorbate-substrate height. Figure 5 shows the whole span of the average adsorbate-substrate height for all possible $(3 \times 3)$ structures. However it is important to notice that the adlayer with a $(3 \times 3)$-sym arrangement is on average the closest one to the substrate. The (3 $\times 3$ )-asym is in the middle of the range. It appears as the intermediate average distance minimum, positioned higher (2.36 in relative units) than the minimum for $(3 \times 3)$-sym adlayer (2.33). In other words, it could be also related to the stability or probability of formation. However, so far we did not find any experimental data to confirm this difference in stability.

In Fig. 6, we illustrate the mechanism of appearance of these two structures by showing the results of a simulation, which involves translation of the $(3 \times 3)$-sym adlayer on the

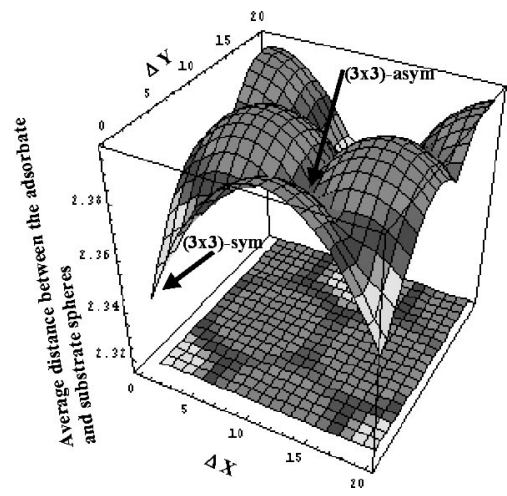

FIG. 6. The change in the average position of the adsorbate layer during continuous translation in $\mathrm{X}$ and $\mathrm{Y}$ directions over the hexagonally ordered substrate, presented in 3D plot.
(111) substrate. Translation was carried out in $\Delta x$ and $\Delta y$ increments, without applying the rotation possibility. As could be clearly seen in this 3D graph, each displacement from the $(3 \times 3)$-sym position (closest to the substrate) results in an increase of the average distance between the adsorbate and substrate. In other words, adlayer atoms are moving away from the surface. The graph also shows that this climbing is not linear. On the way to reach a maximum distance from the substrate, adatoms pass through a local minimum (located at the middle of the surface plot). After taking $X$ and $Y$ coordinates of this minimum and incorporating them in ALSA software, we discovered that adatoms in the minimum are arranged in the $(3 \times 3)$-asym structure. It is important to note that according to the graph presented in Fig. 6, both structures are located at minimum positions, but at different distance from the substrate. This could be easily related to the experimental findings for the iodine adsorption on the $\operatorname{Pt}(111)$ surface, where both structures have been seen frequently. According to our data and the fact that the local minimum for the $(3 \times 3)$-asym structure is located at higher average adsorbate-substrate distance, we believe that the (3 $\times 3$ )-sym structure should be the most stable arrangement for this particular interatomic distance. In order to draw more definitive conclusions about stability of these structures, more theoretical simulations involving estimation of the energy of adsorption and experimental data are necessary.

Furthermore, to understand the relation between all possible adlayer structures we also performed similar analysis for the other two minima seen in the graph in Fig. 3. As shown in Fig. 5, the adlayer with $(\sqrt{3} \times \sqrt{3}) \mathrm{R} 30^{\circ}$ structure could be positioned from the low threefold to the highest atop position. The adlayer with the threefold registry is the closest to the substrate and probably the most stable at ideal conditions. Interestingly, in the whole range of existence of the $(\sqrt{ } 3 \times \sqrt{ } 3) \mathrm{R} 30^{\circ}$ structure, no arrangement with another 
local minima was observed. The span of the adsorbatesubstrate height for $(\sqrt{ } 7 \times \sqrt{ } 7) \mathrm{R} 19.1^{\circ}$ arrangement is more limited and this adlayer could never reach a minimum distance (closeness) to the substrate as $(\sqrt{3} \times \sqrt{3}) \mathrm{R} 30^{\circ}$ adlayer. The range of the average height (distance from the substrate) for $(3 \times 3)$ structures is even more narrow and the $(3 \times 3)$-sym adlayer (the most closest to the substrate from all the $(3 \times 3)$ structures $)$ is on average positioned much further from the substrate layer than the other two structures in their minima. According to results of our simulation, we assume that the adlayer with $(\sqrt{3} \times \sqrt{ } 3) \mathrm{R} 30^{\circ}$ structure, which is on average the closest to the substrate, is the most stable one.

We show that modeling of the unequal-sphere packing could satisfactorily describe all structures of the iodine adlayers on the $\operatorname{Pt}(111)$ surface. However, we do not know if the same model valid for other systems. We preliminary tested our model for identification of the iodine adlayer structures on the $\mathrm{Au}(111)$ surface, which presents nonhexagonal ordered $\mathrm{c}(\mathrm{p} \times \sqrt{ } 3) \mathrm{R} 30^{\circ}$ and shows electrocompression phenomenon, ${ }^{19,45-48}$ and found that another parameter (not an average distance between the adsorbate and substrate layers) should be used for this system description. These preliminary results indicate that one of the crucial steps in our modeling is the definition of the parameter used for selection of the most probable structures among all possible. Further work is in progress to resolve this issue.

\section{CONCLUSION}

In this paper, we introduce a model of unequal-sphere packing based on geometrical principles, which we believe could be applied for atomic level description of the adsorbate-substrate arrangement. As a test of our model, we have searched for the most probable accommodations for an adlayer of bigger spheres laying on the flat substrate with $33 \%$ smaller spheres, both with rigid hexagonal order. The adsorbate layer was allowed to translate and rotate in all the possible directions for an interval of surface coverage. Some of the structures that are formed by these transformations were defined and presented in our paper. With the help of a parameter defined as the average height of the adsorbate layer on the flat substrate, which describes the closeness of the adlayer spheres to the substrate layer, several specific structures have been selected as the most probable: $(\sqrt{ } 3$ $\times \sqrt{ } 3) \mathrm{R} 30^{\circ},(\sqrt{ } 7 \times \sqrt{ } 7) \mathrm{R} 19.1^{\circ}$, and $(3 \times 3)$.

Literature data shows that those structures are typical for the accommodation of the iodine adatoms on the $\mathrm{Pt}(111)$ surface. This verifies, in the best way, our model of the unequal-sphere accommodation with the real adsorbatesubstrate system on an atomic level. In addition, using our model we have been able to explain the origin and translation path of the adsorbate adatoms during formation of the $(3 \times 3)$-asym structure. Although very simple, our approach is sufficient to give a satisfactory model of the iodine arrangement on the $\operatorname{Pt}(111)$ surface.

Although we achieved a very good agreement with real experimental results, our study opens a few questions related to the number of systems which could be explained by such a simple approach.

\section{ACKNOWLEDGMENTS}

The authors wish to gratefully acknowledge support by CONACYT through SNI, UAM-Iztapalapa Division de Ciencia Basica e Ingenieria, and Instituto Mexicano del Petroleo (IMP), Project No. FIES-98-100-I.
${ }^{1}$ G. A. Somorjai, Introduction to Surface Chemistry and Catalysis (Wiley, New York, 1994).

${ }^{2}$ R. I. Masel, Principles of Adsorption and Reaction on Solid Surfaces (Wiley, New York, 1996).

${ }^{3}$ S. N. Magonov and M. H. Whangbo, Surface Analysis with STM and AFM (VCH, Weinheim, 1996).

${ }^{4}$ D. M. Kolb, Physical and Electrochemical Properties of Metal Monolayers on Metallic Substrates (Wiley, New York, 1978), p. 125.

${ }^{5}$ A. T. Hubbard, Chem. Rev. (Washington, D.C.) 88, 636 (1988).

${ }^{6}$ D. M. Kolb, Prog. Surf. Sci. 51, 109 (1996).

${ }^{7}$ A. A. Gewirth and B. K. Niece, Chem. Rev. (Washington, D.C.) 97, 1129 (1997).

${ }^{8}$ K. Itaya, Prog. Surf. Sci. 58, 121 (1998).

${ }^{9}$ O. M. Magnussen, Chem. Rev. (Washington, D.C.) 102, 679 (2002).

${ }^{10}$ W. J. Lorenz and W. Plieth, Electrochemical Nanotechnology (Wiley-VCH, Weinheim, 1996).

${ }^{11}$ D. S. Sholl, A. Asthagiri, and T. D. Power, J. Phys. Chem. B 105, 4771 (2001).

${ }^{12} \mathrm{~S}$. Morita, R. Wiesendanger, and E. Meyer, in Noncontact Atomic Force Microscopy, edited by P. Avouris, K. von Klinzing, H.
Sakaki, and R. Wiesendanger (Springer, Berlin, 2002).

${ }^{13}$ N. P. Lebedeva, M. T. M Koper, J. M. Feliu, and R. A. van Santen, J. Phys. Chem. B 106, 12938 (2002).

${ }^{14}$ Y. Wang and S. J. Sibener, J. Phys. Chem. B 106, 12856 (2002).

${ }^{15}$ T. C. Hales (unpublished).

${ }^{16}$ N. J. A. Sloane, Nature (London) 395, 435 (1998).

${ }^{17}$ S. Torquato, T. M. Truskett, and P. G. Debenedetti, Phys. Rev. Lett. 84, 2064 (2000).

${ }^{18}$ S. C. Chang, S. L. Yau, B. C. Schardt, and M. J. Weaver, J. Phys. Chem. 95, 4787 (1991).

${ }^{19}$ T. Yamada, N. Batina, and K. Itaya, J. Phys. Chem. 99, 8817 (1995).

${ }^{20}$ T. Yamada, K. Ogaki, S. Okubo, and K. Itaya, Surf. Sci. 369, 321 (1996).

${ }^{21}$ T. Yamada, N. Batina, K. Ogaki, S. Okubo, and K. Itaya, in Proceedings of the Sixth International Symposium on Electrode Processes VI, edited by A. Wieckoski and K. Itaya, Electrochem. Soc. 96 (8), 43 (1996).

${ }^{22}$ S. Singh, D. H. Robertson, Q. Peng, and J. J. Breen, Langmuir 13, 5197 (1997).

${ }^{23}$ H. Baltruschat, U. Bringemeier, and R. Vogel, Faraday Discuss. 94, 317 (1982). 
${ }^{24}$ B. C. Schardt, S. L. Yau, and F. Rinaldi, Science 243, 1050 (1989).

${ }^{25}$ S. L. Yau, C. M. Vitus, and B. C. Schardt, J. Am. Chem. Soc. 112, 3677 (1990).

${ }^{26}$ R. Vogel, I. Kamphausen, and H. Baltruschat, J. Phys. Chem. 96, 525 (1992).

${ }^{27}$ R. Vogel and H. Baltruschat, Ultramicroscopy 42, 562 (1992).

${ }^{28}$ N. Shinotsuka, K. Sashikata, and K. Itaya, Surf. Sci. 335, 75 (1995).

${ }^{29}$ K. Itaya, in Electrochemical Nanotechnology, edited by W. J. Lorenz and W. Plieth (Wiley-VCH, Weinheim, 1997), p. 137.

${ }^{30}$ J. Inukai, Y. Osawa, M. Wakisaka, K. Sashikata, Y. G. Kim, and K. Itaya, J. Phys. Chem. B 102, 3498 (1998).

${ }^{31}$ T. E. Felter and A. T. Hubbard, J. Electroanal. Chem. 100, 473 (1979)

${ }^{32}$ G. A. Garwood, Jr. and A. T. Hubbard, Surf. Sci. 92, 617 (1980).

${ }^{33}$ A. Wieckowski, B. C. Schardt, S. D. Rosasco, and J. L. Stickney, Surf. Sci. 146, 115 (1984).

${ }^{34}$ J. L. Stickney, S. D. Rosasco, G. N. Salaita, and A. T. Hubbard, Langmuir 1, 66 (1985).

${ }^{35}$ F. Lu, G. N. Salaita, H. Baltruschat, and A. T. Hubbard, J. Electroanal. Chem. 222, 305 (1987).
${ }^{36}$ M. Saidy, K. A. R. Mitchell, S. A. Furman, M. Labayen, and D. A. Harrington, Surf. Rev. Lett. 6, 871 (1999).

${ }^{37}$ D. G. Frank, N. Batina, T. Golden, and A. T. Hubbard, Science 247, 182 (1990).

${ }^{38}$ H. D. Abruna, in X-Ray Absorption Spectroscopy in the Study of Electrochemical Systems, edited by H. D. Abruna (VCH Publishers, New York, 1991).

${ }^{39}$ R. M. Corn, Proc. Electrochem. Soc. 11, 264 (1992).

${ }^{40}$ S. K. Jo and J. M. White, Surf. Sci. 261, 111 (1992).

${ }^{41}$ D. G. Frank, O. M. R. Chyan, T. Golden, and A. T. Hubbard, J. Phys. Chem. 97, 3829 (1993).

${ }^{42}$ D. G. Frank, O. M. R. Chyan, T. Golden, and A. T. Hubbard, J. Phys. Chem. 98, 1895 (1994).

${ }^{43}$ B. M. Ocko, O. M. Magnussen, J. X. Wang, and R. R. Adzic, Physica B 221, 238 (1996).

${ }^{44}$ C. A. Lucas, N. M. Markovic, and P. N. Ross, Phys. Rev. B 55, 7964 (1997).

${ }^{45}$ X. Gao and M. J. Weaver, J. Am. Chem. Soc. 114, 8544 (1992).

${ }^{46}$ B. M. Ocko, G. M. Watson, and J. Wang, J. Phys. Chem. 98, 897 (1994).

${ }^{47}$ N. Batina, T. Yamada, and K. Itaya, Langmuir 11, 4568 (1995).

${ }^{48}$ T. Yamada, N. Batina, and K. Itaya, Surf. Sci. 335, 204 (1995). 